\section{Ulmer Heft II}

Das 20-jährige Jubiläum der Ulmer Universitätsklinik für Psychiatrie hat uns $\mathrm{zu}$ zwei Ulmer Heften motiviert. Auch in diesem zweiten Heft stellen wir Artikel zu aktuellen Forschungsthemen aus unserem Haus vor.

Tatjana Meier und Birgit Abler beschäftigen sich mit der serotonergen Modulation der Schmerzwahrnehmung. Sowohl tierexperimentelle Studien als auch Humanexperimente sowie klinische Beobachtungen weisen darauf hin, dass neben dem Endorphinsystem das serotonerge Transmittersystem bei der Wahrnehmung und Verarbeitung von Schmerzen eine zentrale Rolle spielt. Dabei gilt es zwischen akutem und chronischem Schmerz zu differenzieren sowie zwischen nozizeptivem und neuropathischem Schmerz. Zu diesem Artikel stellen wir Ihnen 10 CME-Fragen zur Verfügung, die Sie zum Erwerb von Fortbildungspunkten bearbeiten können.

In ihrem Beitrag stellen Alexander Karabatsiakis und Carlos Schönfeldt-Lecuona eine neue Hypothese zur Pathophysiologie der Depression auf. Im Fokus stehen die Mitochondrien, die die Zellen mit Energie in Form von ATP versorgen. Neue labortechnische Verfahren ermöglichen es, die Integrität der Mitochondrien in den Zellen $\mathrm{zu}$ charakterisieren. Die Autoren stellen die mögliche Beteiligung der Mitochondrien bei der Entstehung der Depression dar und diskutieren einen möglichen mitochondrialen Wirkmechanismus der Elektrokonvulsionstherapie.

In zwei Beiträgen steht eine psychotherapeutische Methode, das sogenannte Imagery Rescripting, im Mittelpunkt. Hierbei geht es um das Abrufen, Interpretieren und Neu-Bewerten mentaler Bilder. Nicht nur verbalisierte Gedanken, sondern auch sich aufdrängende Bilder vor dem geistigen Augen spielen bei einer Reihe von psychischen Störungen eine Rolle. Die Nutzung dieser mentalen Bilder zur Therapie psychischer Störungen hat eine lange komplexe Geschichte. Auch die kognitive Verhaltenstherapie hat das Imaginieren in ihr Repertoire aufgenommen und die Bearbeitung in manualisierter Form etabliert. Anna Maier und Mitarbeiter stellen den Einsatz dieser Methode bei der Behandlung von Angststörungen vor. Caroline Schaitz und Mitarbeiter beschäftigen sich in ihrem Beitrag mit den mentalen Bildern, die von Patienten im Rahmen einer BorderlinePersönlichkeitsstörung berichtet werden. In einer Studie untersuchten sie den $\mathrm{Zu}$ sammenhang zwischen selbstschädigendem Verhalten und den dazu berichteten mentalen Bildern, was möglicherweise zur Entwicklung neuer Therapieverfahren beitragen könnte.

Julia Kröner und Mitarbeiter beschäftigen sich mit dem physiologischen endokrinen Stresssystem und den Veränderungen, die bei Patienten mit Borderline-Persönlichkeitsstörung beobachtet werden konnten. Zu der Hypothese einer spezifisch hochregulierten Stressreaktion bei den betroffenen Patienten stellen sie die aktuelle Befundlage in der Literatur zusammen. Hierbei stehen interpersoneller und sozial-evaluativer Stress im Vordergrund.

Schließlich präsentieren Markus Kölle und Mitarbeiter einen interessanten Fall aus unserer ADHS-Ambulanz. Es handelt sich um einen Patienten mit gesicherter multipler Sklerose, bei dem zusätzlich ein ADHS diagnostiziert und erfolgreich mit Stimulanzien behandelt wurde.

Thomas Kammer \& Manfred Spitzer, UIm

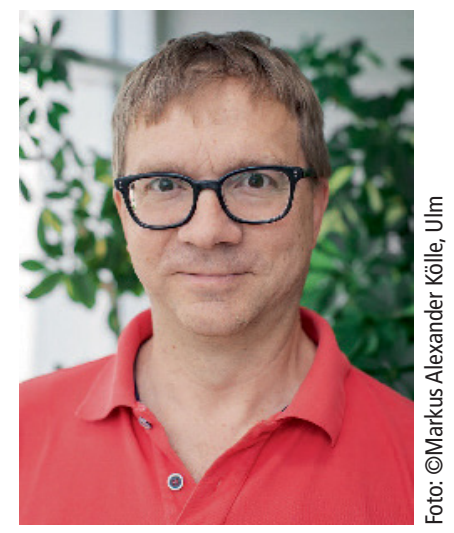

Prof. Dr. Thomas Kammer, Sektion für Neurostimulation, Klinik für Psychiatrie und Psychotherapie III, Universität UIm

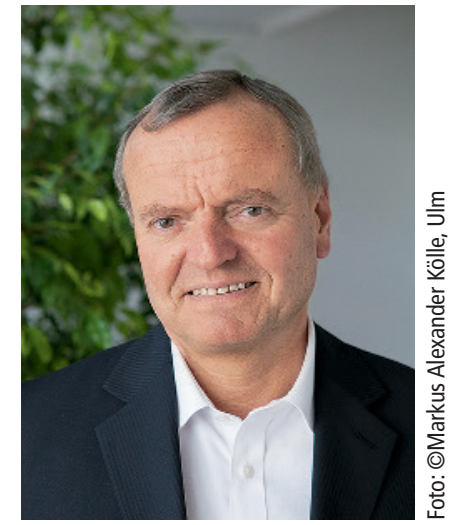

Prof. Dr. Dr. Manfred Spitzer, Klinik für Psychiatrie und Psychotherapie III, Universität Ulm 